\title{
Light harvesting in photosystem II
}

\author{
Herbert van Amerongen · Roberta Croce
}

Received: 27 January 2013/Accepted: 8 April 2013/Published online: 18 April 2013

(c) The Author(s) 2013. This article is published with open access at Springerlink.com

\begin{abstract}
Water oxidation in photosynthesis takes place in photosystem II (PSII). This photosystem is built around a reaction center (RC) where sunlight-induced charge separation occurs. This RC consists of various polypeptides that bind only a few chromophores or pigments, next to several other cofactors. It can handle far more photons than the ones absorbed by its own pigments and therefore, additional excitations are provided by the surrounding light-harvesting complexes or antennae. The RC is located in the PSII core that also contains the inner light-harvesting complexes CP43 and CP47, harboring 13 and 16 chlorophyll pigments, respectively. The core is surrounded by outer light-harvesting complexes (Lhcs), together forming the so-called supercomplexes, at least in plants. These PSII supercomplexes are complemented by some "extra" Lhcs, but their exact location in the thylakoid membrane is unknown. The whole system consists of many subunits and appears to be modular, i.e., both its composition and organization depend on environmental conditions, especially on the quality and intensity of the light. In this review, we will provide a short overview of the relation between the structure and organization of pigment-protein complexes in PSII, ranging from individual complexes to entire membranes and experimental and theoretical results on excitation energy transfer and charge separation. It will become clear that time-resolved fluorescence data can
\end{abstract}

H. van Amerongen ( $\square)$

Laboratory of Biophysics, Wageningen University,

P. O. Box 8128, 6700 ET Wageningen, The Netherlands

e-mail: herbert.vanamerongen@wur.nl

R. Croce

Department of Physics and Astronomy, Faculty of Sciences,

VU University Amsterdam, De Boelelaan 1081,

1081 HV Amsterdam, The Netherlands provide invaluable information about the organization and functioning of thylakoid membranes. At the end, an overview will be given of unanswered questions that should be addressed in the near future.

Keywords Excitation energy transfer - Picosecond fluorescence - Thylakoid membrane - Charge separation . State transitions

\section{Introduction}

The photosynthetic light reactions of green plants, algae, and cyanobacteria take place in photosystems I and II (PSI and PSII). Light-induced charge separation in the reaction center (RC) of PSII leads to the oxidation of water, the reduction of plastoquinone and the formation of a proton gradient across the thylakoid membrane in which PSI and PSII are embedded, which is crucial for the production of ATP. PSII and PSI work in series and together they also drive $\mathrm{NADP}^{+}$to NADPH reduction with $\mathrm{H}_{2} \mathrm{O}$ as electron donor (Nelson and Yocum 2006). Light-induced charge separation in the RC of PSII starts from the primary donor P680 and an electron proceeds via a pheophytin onto plastoquinone $Q_{\mathrm{A}}$ and subsequently to plastoquinone $Q_{\mathrm{B}}$. The primary cation radical $\mathrm{P} 680^{+}$has an $E_{\mathrm{m}}$ value of $+1.25 \mathrm{~V}$ (Rappaport et al. 2009), far higher than the value of +0.80 for Chl in solution (Kobayashi et al. 2007) and this high value is ultimately responsible for the oxidation of water.

The RC of PSII itself only contains six chlorophylls $a$ (Chls $a$ ) and two pheophytins but it is always present in the so-called core complex that also contains the pigmentproteins CP43 and CP47, providing additional 13 and 16 Chls $a$, respectively, together with several $\beta$-carotene 
molecules (see (Umena et al. 2011) for the most recent PSII core structure). Both antenna complexes feed excitation energy into the RC. These antenna Chls are on the one hand at a "safe" distance from the RC pigments, which are highly oxidizing after charge separation (see Fig. 1), preventing direct pigment oxidation in the antenna, and on the other hand close enough to perform efficient excitation energy transfer (EET).

The core consists of $\sim 20$ different subunits, and the pigment/protein ratio is low which makes it a rather expensive piece of machinery. To increase the absorption cross-section further in a cost-effective way, additional light-harvesting complexes have appeared during evolution. Plants and green algae possess membrane-embedded antennae with a high pigment/protein ratio $(\sim 1: 2$ regarding the mass), also called outer light-harvesting complexes (Lhcs) and to a large extent they are organized in larger supercomplexes, together with the PSII cores. Cyanobacteria, that appeared earlier in evolution contain membraneassociated phycobilisomes (see e.g., (Neilson and Durnford 2010)) with a pigment-to-protein ratio that is substantially lower $(\sim 1: 5)$ although still higher than for the core complex. For recent studies of EET in/from phycobilisomes in vitro and in vivo the reader is referred to Tian et al. (Tian et al. 2011, 2012). The present review will focus on light harvesting in plants.

The thylakoid membrane in plants is divided into grana, which are composed of stacks of membrane disks, and stroma lamellae, which connect the various grana in the choroplast (Mustardy and Garab 2003; Shimoni et al. 2005; Mustardy et al. 2008; Daum et al. 2010; Kouril et al. 2011). PSII is located in the grana (Andersson and Anderson 1980) whereas PSI is mainly present in the stroma lamellae (together with the ATP synthase). The thylakoid membrane is flexible and dynamic and able to respond to changes in environmental conditions by changing both composition and organization of the PSII supercomplexes (Anderson et al. 2008; Chuartzman et al. 2008; Goral et al. 2010). It has been shown that part of the grana membrane contains PSII arrays that consist of supercomplexes with different antenna sizes, but the abundance of the arrays seems to depend on the composition of PSII which for instance depends on the species analyzed and on the growth conditions (Boekema et al. 2000; Kouril et al.; Daum et al. 2010; Kirchhoff et al. 2007; Kouril et al. 2012; Kiss et al. 2008) (Kereiche et al. 2010; Kovacs et al. 2006; de Bianchi et al. 2008).

Only part of the PSII supercomplexes is embedded in these regular arrays, while another part is less organized. It
Fig. 1 Chlorophyll organization in the core complex of PSII (Guskov et al. 2009). Chls P, red; Chls D1 and $\mathrm{D} 2$, orange; Chls z green; Pheos, yellow. The Chls of CP47 are in blue and those of CP43 in cyan. The phytol chains of the Chls are omitted for clarity. The upper figure shows a top view (from the stroma) and the lower figure provides a side view
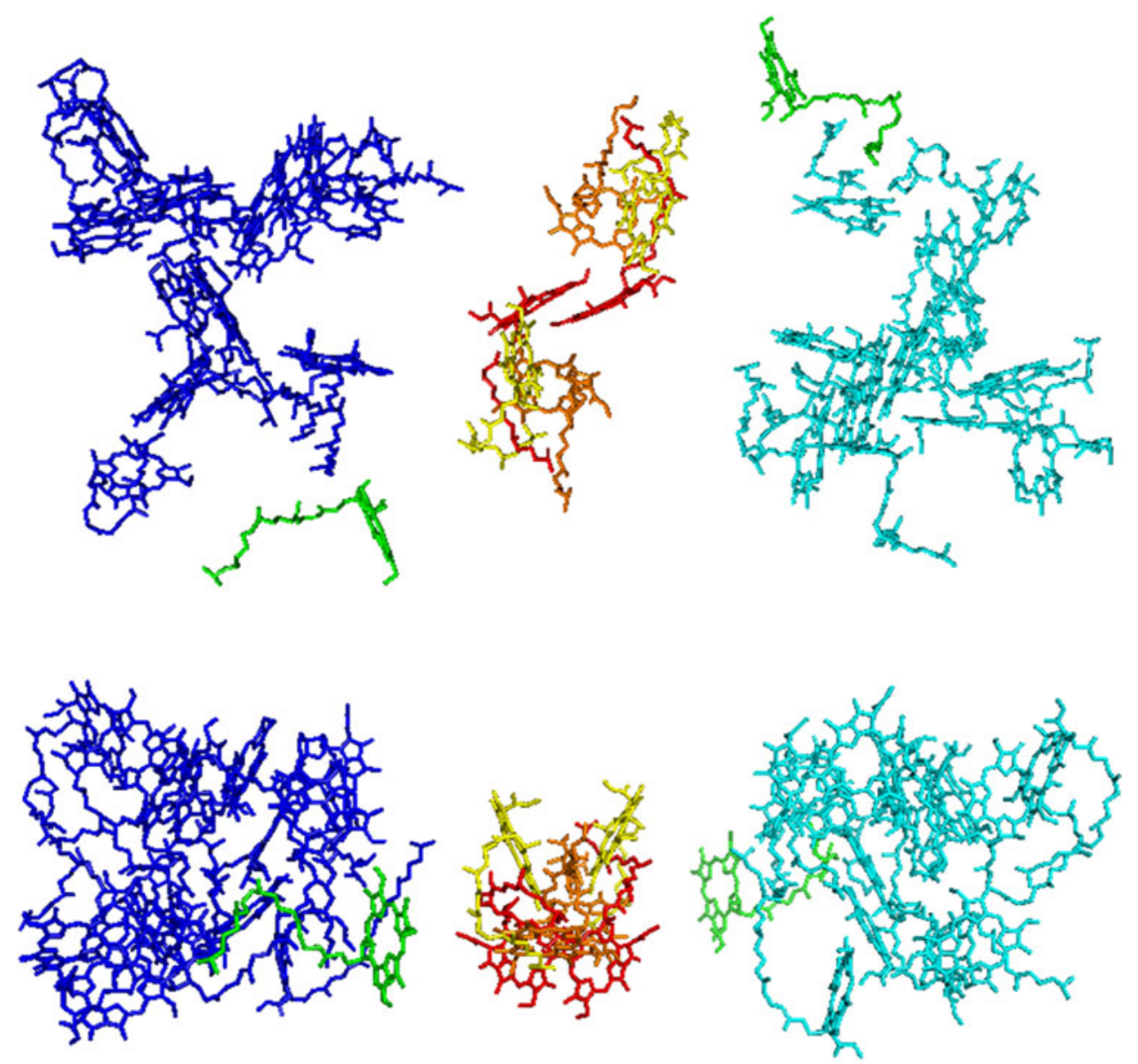
is not exactly clear yet what the role of the arrays and the other parts is. But it is known that reorganizations in both arrays and other parts take place as a function of light quality and intensity (Wientjes et al. 2013; Kouril et al. 2012; Jahns and Holzwarth 2012; Betterle et al. 2009).

In Fig. 2, a model of a plant PSII supercomplex is shown. It is composed of a PSII core together with the gene products of genes Lhcb1-6 in a well-defined arrangement. The largest supercomplexes contain a dimeric core, four LHCII (encoded by Lhcb1-3) trimers, two strongly bound (S) and two moderately strongly bound (M), and two monomeric copies each of CP29 (Lhcb4), CP26 (Lhcb5), and CP24 (Lhcb6). Supercomplexes of different sizes can be isolated (Caffarri et al. 2009), which is probably partly due to the solubilization process but it is also known that a sub-population of smaller supercomplexes is also observed in high light plants (see e.g., (Daum et al. 2010; Kouril et al. 2012)).

The Lhc complexes are densely packed with Chl $a$ and $b$ pigments and the xanthophylls lutein (Lut), violaxanthin $(\mathrm{Vx})$, and neoxanthin (Nx) (with the exception of CP24 that

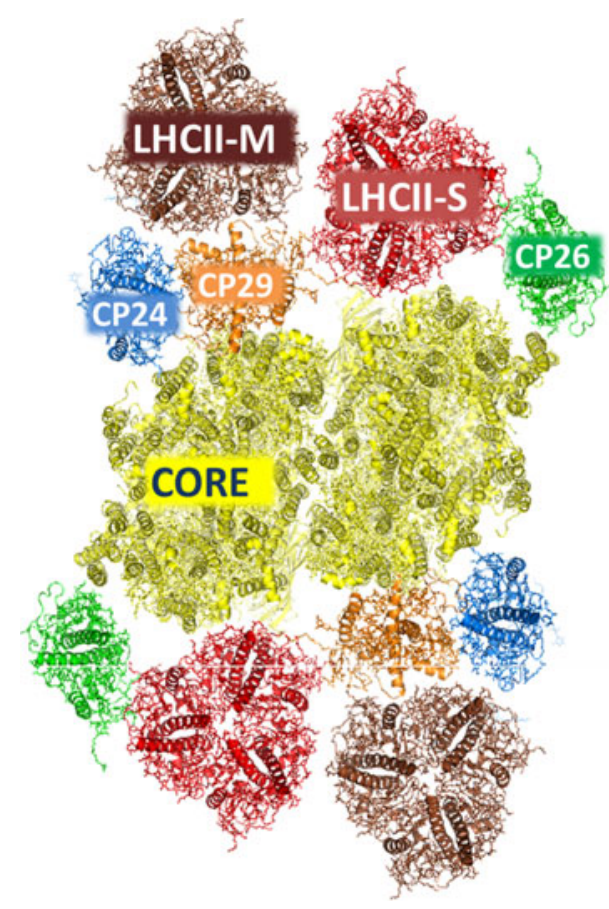

Fig. 2 Model of the PSII supercomplex C2S2M2 from higher plants. Top-view for the stromal side on a C2S2M2 supercomplex from $A$. thaliana. Based on the projection map of $\mathrm{C} 2 \mathrm{~S} 2 \mathrm{M} 2$ at $12 \AA$ resolution, a model of the 3D structure of the supercomplex was reconstructed (Caffarri et al. 2009) using the crystal structures of PSII core (Guskov et al. 2009) and LHCII (Liu et al. 2004). For the minor antenna complexes, the structure of a monomer of LHCII was used while the pigment composition/occupancy was assigned based on the results of mutation analysis experiments on in vitro reconstituted complexes (Bassi et al. 1999; Remelli et al. 1999; Ballottari et al. 2009; Passarini et al. 2009) does not contain $\mathrm{Nx}$ ) which are responsible for light absorption and EET. Xanthophyll excitations (xanthophylls are carotenoids which contain oxygen) are rapidly transferred, typically within one ps to the Chls that are in Van der Waals contact with these carotenoids. Chls $b$ transfer excitations to Chls $a$, which have lower excited-state energy, and on average only a small fraction of the excitations $(\sim 5 \%)$ is located on Chl $b$ molecules, due to Boltzmann equilibration in the excited state. Via rapid EET between mainly Chls $a$ the excitations end up in the RC (see (Croce and van Amerongen 2011) for a review). Some of the Chl $a$ singlet excitations are transformed into $\mathrm{Chl}$ $a$ triplets, which can lead to the formation of destructive singlet oxygen molecules. Fortunately, most of these dangerous Chl triplets $(>95 \%)$ are scavenged by the carotenoids that are in Van der Waals contact with Chl $a$ (Barzda et al. 1998; Lampoura et al. 2002; Mozzo et al. 2008a; Carbonera et al. 1992; van der Vos et al. 1991).

In this review, we will focus on the study of EET and CS in PSII, starting with the core, followed by outer antenna complexes and supercomplexes. A brief overview will then be given of results on thylakoid membranes, isolated from plants with varying antenna composition as a result of short- and long-term differences in light conditions. At the end, some unsolved problems will be presented together with suggestions for further research. We would also like to refer to other reviews from recent years for further information (Renger and Schlodder 2010; Vassiliev and Bruce 2008; Renger 2010; Van Amerongen et al. 2003; Minagawa and Takahashi 2004; Barber 2002; Muh et al. 2008; Renger and Renger 2008; Croce and van Amerongen 2011).

\section{The PSII core}

In Fig. 3, the reconstructed picosecond fluorescence kinetics of the PSII core from Thermosynechococcus from two different studies are shown (Miloslavina et al. 2006; van der Weij-de Wit et al. 2011) and the results are nearly identical. Accurate data fitting requires five or more exponentials but two direct observations stand out. Charge separation occurs with an average time constant $\tau$ below $100 \mathrm{ps}$, leading to the relatively fast disappearance of the (fluorescence) signal. Since this time constant is much shorter than the normal excited-state lifetime of chlorophyll (typically several ns), this guarantees a high quantum efficiency of charge separation $\left(\phi_{\mathrm{CS}}\right)$. The corresponding value is above 0.95 , using the well-known relation $\phi_{\mathrm{CS}}=$ $1-\tau / \tau_{\mathrm{Chl}}$ (Croce and van Amerongen 2011), where $\tau_{\mathrm{Chl}}$ is the average lifetime of the excited $\mathrm{Chl}$ in PSII in the absence of charge separation. The exact value for this parameter is unknown but a recent study led to a value of 
$\sim 2$ ns (Belgio et al. 2012). The kinetics also shows a small contribution of a long-lived component which is usually ascribed to the fact that charge separation is partly reversible. The amplitude and lifetime of this component depend on the competition between secondary charge separation in the RC (forward electron transfer from the primary electron acceptor) and back transfer of the electron from primary acceptor to primary donor.

Although the kinetics in both studies is rather similar, the models that were used for the fitting differ considerably. It should be noted that the overall (average) trapping time $\tau$ of excitations can in good approximation be considered as the sum of two terms: $\tau=\tau_{\text {mig }}+\tau_{\text {trap }}$ (Van Amerongen et al. 2000; Broess et al. 2006). In a traplimited model, the equilibration time (also called migration time $\tau_{\mathrm{mig}}$ ) of excitations over the photosystem is assumed to be much shorter than the overall trapping time, i.e., it can largely be neglected and thus $\tau=\tau_{\text {trap. }}$. The bestknown trap-limited model is the so-called exciton/radical pair equilibrium model (ERPE model) (van Grondelle 1985; Schatz et al. 1988, 1987), and it has widely been used to interpret all kinds of variations in fluorescence in photosynthesis. Besides primary charge separation, it also includes charge recombination and secondary charge separation (see above). In (Miloslavina et al. 2006), the data were fitted to a kind of trap-limited model and it was thus

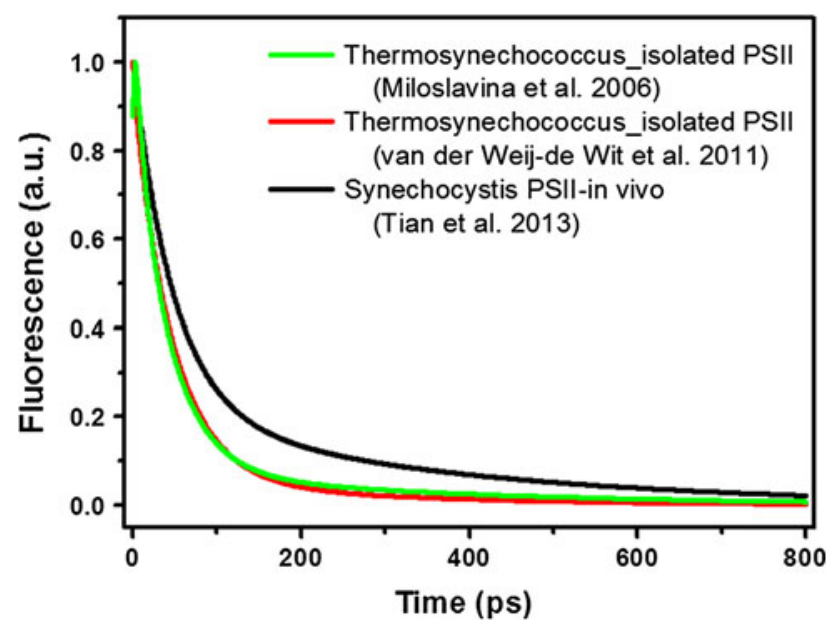

Fig. 3 Picosecond kinetics of isolated PSII core complexes from Thermosynechococcus, reconstructed from (Miloslavina et al. 2006) (black solid) and (van der Weij-de Wit et al. 2011). The decay curve presented in (Miloslavina et al. 2006) was reconstructed based on the DAS shown in Fig. 7 of that work, and only $\tau 1-\tau 5$ are included in the calculation. The decay curve from (van der Weij-de Wit et al. 2011) was reconstructed based on the compartmental scheme shown in Fig. 6 in that article and the initial excitation fractions therein. Excitation wave lengths were 663 and $400 \mathrm{~nm}$, respectively, but these differences are not expected to significantly influence the overall kinetics. The dotted line represents the fluorescence kinetics of PSII core in vivo for a Synechocystis mutant (excitation wavelength $400 \mathrm{~nm}$ ) (Tian et al. 2013) assumed that excitation equilibration in the core occurs on a time scale much faster than the overall trapping time. In good approximation $\tau_{\text {trap }}$ is equal to the intrinsic charge separation time of the primary donor $\tau_{\mathrm{iCS}}$, divided by the probability that the excitation is actually located on the primary donor. For $N$ isoenergetic pigments, including the primary donor, $\tau_{\text {trap }}=N \tau_{\text {iCs }}$ (when charge recombination is ignored). Taking for instance values of $\tau_{\text {trap }}=60 \mathrm{ps}$ and $N=35$, one finds that $\tau_{\mathrm{iCS}}=1.7 \mathrm{ps}$.

However, the distances between the pigments in these complexes and the ones in the RC (Fig. 1) are so large that it was concluded in (van der Weij-de Wit et al. 2011) that the transfer time of excitations to the trap and therefore the contribution of $\tau_{\mathrm{mig}}$ cannot be ignored. This means that the value of $\tau_{\text {trap }}$ should be smaller and concomitantly the same should be true for $\tau_{\mathrm{iCS}}$, which also comes out of the fitting (van der Weij-de Wit et al. 2011). Very recently, the picosecond fluorescence kinetics was obtained for the PSII core in vivo, by comparing the results of different mutants of Synechocystis PCC 6803 mutants (Tian et al. 2013). It turned out that the PSII core of this organism in vivo was somewhat slower than the one of Thermosynechococcus in vitro but again, the kinetics could be satisfactorily fitted with both a trap-limited and a migration-limited model. It is clear that comparing different fitting models cannot favor one trapping model above the other.

In a recent theoretical treatment Raszewski and Renger (Raszewski and Renger 2008) concluded that the trapping should be migration-limited: Transfer from CP43/CP47 occurs with time constants of 40-50 ps. The main reason for the slow transfer is the large distance between the pigments in the core antenna and those in the RC. As was mentioned above, this large distance is probably needed to avoid oxidation of the antenna pigments. The consequence of this slow EET is that the primary charge transfer time should be extremely fast, i.e., around $300 \mathrm{fs}$, accompanied by a very large initial drop in free energy to explain the overall timeresolved results. It should be noted that at least in isolated $\mathrm{RC}$ complexes such a fast charge separation time was not observed (Groot et al. 2005; Germano et al. 2004; van Mourik et al. 2004; Holzwarth et al. 2006; Prokhorenko and Holzwarth 2000; Andrizhiyevskaya et al. 2004; Wasielewski et al. 1990; Durrant et al. 1992; Pawlowicz et al. 2008) and one might wonder whether this is realistic. On the other hand, it is possible that isolated RC complexes are "slower" than the ones in vivo (see also below).

It is worthwhile to mention that the average lifetimes of core preparations from cyanobacteria are in general far shorter than for cores from plants (Raszewski and Renger 2008). Although this may be due to differences in the intrinsic properties of the cores, it is most likely related to problems associated with the isolation of core preparations from plants. 
At the moment, there are still several unsolved issues with respect to PSII core kinetics. Both trap- and migration-limited models seem to have some intrinsic problem and maybe we should consider the possibility of coherent EET into the RC (Collini and Scholes 2009). Moreover, we do not know to which extent observed differences between in vivo and in vitro results are due to differences in biological species or to the fact that the thylakoid membrane might influence the primary EET and CT steps. Why do cores isolated from plants show slower kinetics than those of cyanobacteria? We have not mentioned any studies on the kinetics of isolated RCs but as was discussed in (Broess et al. 2006), they appear to be substantially slower than when embedded in larger systems. Therefore, it seems that the study of isolated complexes at the moment can only contribute to basic knowledge about charge separation mechanisms and pathways in PSII but they do not give realistic time constants.

\section{Outer antenna complexes}

The antenna complexes of PSII from higher plants are composed of members of the Lhc multigenic family. The structure of a monomeric subunit of trimeric LHCII (Liu et al. 2004; Standfuss et al. 2005) is given in Fig 4. Each monomer coordinates eight Chls $a$, six Chls $b$ and four xanthophylls (one Nx, two Lut's and one Vx). The two Lut's are located at sites L1 and L2 in the center of the molecule while $\mathrm{Nx}$ and $\mathrm{Vx}$ are located at the periphery in sites N1 and V1, respectively (Croce et al. 1999; Caffarri et al. 2001; Ruban and Horton 1999). The average distance between the Chls is around $10 \AA$, which leads to excitonic interactions between the pigments, resulting in fast energy transfer within the complex.

Based on sequence similarity, all members of the Lhc family are thought to have a similar structural arrangement (Green and Khlbrandt 1995) and most of the amino acids that bind $\mathrm{Chl}$ in LHCII are conserved in all family members (Bassi et al. 1997). This is now confirmed for CP29 and Lhca1-4 based on the comparison of the structures (Pan et al. 2011; Amunts et al. 2010). The structures of CP29 and monomeric LHCII are shown in Fig. 4. Nevertheless, individual complexes show different biochemical and spectroscopic properties (see e.g., (Pascal et al. 1999)), mainly due to the fact that the pigment composition is not identical (Sandona et al. 1998). Mutations of the putative Chl-binding residues followed by in vitro reconstitution (Plumley and Schmidt 1987) has allowed the characterization of the chromophores in most binding sites (Bassi et al. 1999; Remelli et al. 1999; Yang et al. 1999; Rogl and Kuhlbrandt 1999; Ballottari et al. 2009; Passarini et al. 2009). The four Chl-binding sites in the center (602, 603,
610 and 612) accommodate Chls $a$ in all antenna complexes. Chls 602-603 absorb around $675 \mathrm{~nm}$ and Chls 610-612 absorb around $680 \mathrm{~nm}$, representing the lowest energy state(s) of the system (Remelli et al. 1999; Rogl and Kuhlbrandt 1999). The domain including helix C mainly coordinates Chls $b$ (Remelli et al. 1999; Peterman et al. 1996). In all complexes, site L1 contains a Lut while L2 accommodates Lut in LHCII and CP26 but Vx in CP29 and $\mathrm{CP} 24$. Nx is present in the $\mathrm{N} 1$ site of all complexes apart from CP24 (Caffarri et al. 2007).

By combining the results of a large number of different studies on LHCII in the nineties (Visser et al. 1996; Savikhin et al. 1994a; Peterman et al. 1997; Croce et al. 2001; Connelly et al. 1997), it was concluded that (sub)picosecond EET leads to ps spectral equilibration and excitations become mainly localized on the peripheral Chl $a$ pigments on the stromal part of the protein i.e., Chls 610-612 (Van Amerongen and van Grondelle 2001). From there, they can be transferred to neighboring complexes in the thylakoid membrane. Spatial equilibration within the trimers occurs on a slower time scale (tens of ps) as was concluded from several other studies (Savikhin et al. 1994b; Barzda et al. 2001; van Oort et al. 2007; Kwa et al. 1992; Novoderezhkin and van Grondelle 2010).

The results of the various time-resolved and steady-state spectroscopic studies were later modeled with the use of Redfield theory (Novoderezhkin et al. 2004, 2005) and led to a theoretical description of the data largely consistent with the crystal structure (Liu et al. 2004; Standfuss et al. 2005), demonstrating that within a few ps, the excitations are mainly localized on Chls 610-612.

More recent studies using 2-D electronic spectroscopy (Calhoun et al. 2009) are at least qualitatively in agreement with the modeling results of Novoderezhkin et al. (Novoderezhkin et al. 2005; Novoderezhkin and van Grondelle 2010) although it is not known whether the new models also lead to a correct description of for instance the lineardichroism (LD) (Van Amerongen et al. 1994) and circulardichroism (CD) spectra (Georgakopoulou et al. 2007). It is worth to point that in a very recent study, Müh and Renger were able to obtain rather satisfactory fits of all steady-state spectra of LHCII, demonstrating that not all site energies agree with those obtained before and that also the absolute LD spectra do not perfectly agree with the crystal structure (Muh and Renger 2012). Therefore, it seems that there is room for an additional round of improving both the structural model of LHCII and the understanding of its steady-state and time-resolved spectroscopic properties. At this point, it is also worth to mention that Zucchelli et al. (Zucchelli et al. 2012) recently calculated LHCII absorption spectra and obtained substantial variation for the monomeric subunits of three different trimers taken from the crystal structure (Liu et al. 2004; Standfuss et al. 2005), 


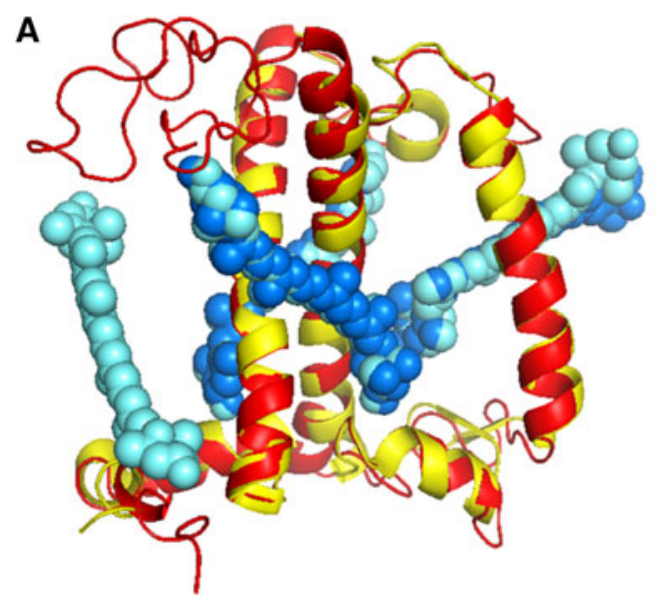

Fig. 4 Overlap of the structural models of LHCII (Liu et al. 2004) and CP29 ((Pan et al. 2011)). a Side view (from within the membrane) on the protein backbone of LHCII (red) and CP29 (yellow) and the xanthophylls of LHCII (light blue) and CP29 (dark blue). Main differences are the lack of the $N$-terminal part of CP29 which

due to variations in the conformation of the Chl macrocycle and variations in the excitonic coupling strength between different Chls. Finally, it is worth mentioning that the (sub)ps transient absorption kinetics of the three gene products forming LHCII, Lhcb1, Lhcb2, and Lhcb3, are identical (Palacios et al. 2006).

EET in the minor antenna complexes (Cinque et al. 2000; Gradinaru et al. 1998, 2000; Salverda et al. 2003; Croce et al. 2003a, b; Marin et al. 2010, 2011) seems to occur along similar pathways as in LHCII. Also in these complexes equilibration occurs within a few ps, leading to excitation population mainly on Chls $610-612$, the lowest energy pigments located on the stromal side at the periphery of the complex (Mozzo et al. 2008b).

\section{PSII supercomplexes}

Obtaining homogeneous preparations of PSII supercomplexes is difficult because they disassemble quite easily (Wientjes et al. 2009; Caffarri et al. 2001). The largest supercomplex purified so far is C2S2M2 (Fig. 2) (Caffarri et al. 2009) and it is the most abundant complex in thylakoid membranes of Arabidopsis thaliana (Dekker and Boekema 2005; Kouril et al. 2012). The LHCII trimers differ somewhat in composition. The $\mathrm{S}$ trimer is composed of the products of the Lhcb1 and Lhcb2 genes and the M trimer in addition also contains the product of the Lhcb3 gene (Hankamer et al. 1997). Ordered arrays of C2S2, C2S2M, and C2S2M2 have been observed in membranes of different plants (Boekema et al. 2000; Daum et al. 2010; Yakushevska et al. 2001; Kouril et al. 2011). Smaller supercomplexes have also been purified but they are probably

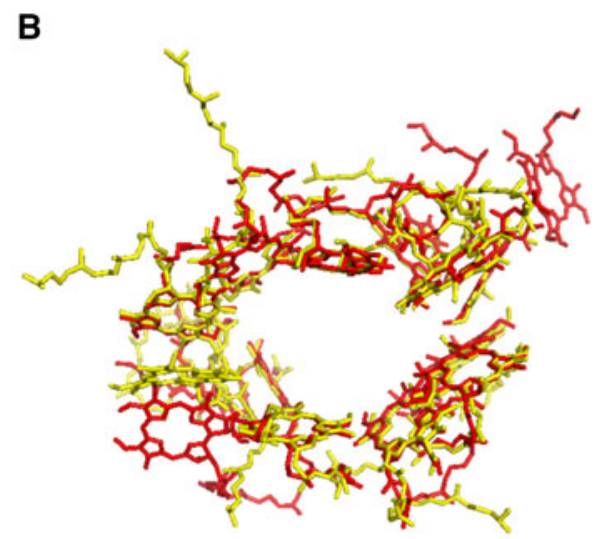

apparently was cleaved off during crystallization and the lack of VX in CP29. For the rest, both proteins show very similar structures. b Top view showing that the Chl organization in LHCII (red) and CP29 (yellow) is rather similar although not identical

partly disassembled (Caffarri et al. 2009). Based on a projection map of the $\mathrm{C} 2 \mathrm{~S} 2 \mathrm{M} 2$ supercomplex at $12 \AA$ resolution (Caffarri et al. 2009) and the crystal structures of core and LHCII, a 3D supercomplex structure has been reconstructed (Fig. 2). Such a model can be used to visualize possible EET pathways (Croce and van Amerongen 2011).

Picosecond fluorescence measurements have been performed on four different PSII supercomplex preparations from A. thaliana (Caffarri et al. 2011). The smallest complex (C2S) contains a dimeric PSII core plus CP26, CP29 and one LHCII trimer. The largest complex (C2S2M2) corresponds to the structure in Fig. 2. The average fluorescence lifetime becomes longer upon increasing the antenna size from $109 \mathrm{ps}$ for the dimeric core complex $(\sim 70 \mathrm{Chl}$ $a$ molecules) to $158 \mathrm{ps}$ for $\mathrm{C} 2 \mathrm{~S} 2 \mathrm{M} 2(\sim 210 \mathrm{Chl} a$ molecules), using a detergent concentration of $0.01 \% \alpha$-DM. In $0.001 \% \alpha$-DM the lifetimes decrease on average by around 20 ps. Plotting the average lifetimes versus the number of Chls $a$ for the four supercomplex preparations and the core, shows that all values lie more or less on a straight line which evidently is not going through the origin as one might expect (Van Amerongen et al. 2003) indicating that the energy transfer and/or charge separation rates are reduced in complexes with decreased size, strongly suggesting that the antenna system is important for plant PSII integrity and functionality, which is also supported by biochemical results (Caffarri et al. 2011).

The kinetics were also simulated using coarse-grained modeling and the obtained parameters were used to illustrate various aspects of PSII functioning (Caffarri et al. 2011). It was for instance calculated that for the largest supercomplex the efficiency of charge separation is $89 \%$. In the presence of one open and one closed RC, the 
photochemical efficiency reduces to $78 \%$, which is much larger than the value of $45 \%$ calculated when the cores are not connected into dimers. This demonstrates that a dimeric conformation increases the light-harvesting capacity by more than $70 \%$ in the presence of one closed RC. This is an important property for PSII because of its slow turnover and it also suggests that the arrays of PSII that are observed in electron-microscopy measurements are advantageous when a substantial fraction of the RC's is closed.

In fact, the advantage of PSII units being connected to each other was already discussed many decades ago and it was experimentally determined that indeed many "photosynthetic units" (PSU's) are connected to each other (see e.g., (Clayton 1981)). Two popular models from those days were the puddle model, in which PSU's were not connected and the lake model, in which basically all PSU's were connected. Whereas for purple bacteria, the lake model is applicable, it was found that for plants, the situation was somewhere in between these extreme models (see e.g., also (Clayton 1981)), which is in agreement with the organization observed with electron-microscopy (see above).

\section{Energy transfer and charge separation in PSII membranes}

Grana membranes can be purified (the so-called BBY particles) that contain practically only PSII complexes (Berthold et al. 1981; Dunahay et al. 1984; Albertsson et al. 1981), although it is not completely understood how PSII is organized in these membranes. It had been suggested that $\mathrm{C} 2 \mathrm{~S} 2$ represents the supercomplex in high light, while $\mathrm{C} 2 \mathrm{~S} 2 \mathrm{M} 2$ is the result of low-light growth (Daum et al. 2010). However, it was recently demonstrated that also in high light, C2S2M2 is still the main supercomplex in Arabidopsis (Kouril et al. 2012). In high light, the amount of LHCII trimers is lower than in low light, although in all cases the stoichiometry LHCII/core is higher than two (it is often between three and four) (Bailey et al. 2001; Anderson and Andersson 1988; Kouril et al. 2012), meaning that not all LHCII trimers are present in the supercomplexes but that there are also "extra" trimers. The location of these "extra" LHCII trimers, however, is still unknown and some of them might be located in the LHCII-only domains that were proposed by Boekema et al. (Boekema et al. 2000) although it should be emphasized that most of the "extra trimers" should be connected to PSII which is not necessarily the case for these LHCII-only domains. Part of these extra trimers attaches to PSI in the presence of light but detaches again in the dark and also during light-stress conditions (Wientjes et al. 2013). Recently, it was also found that in Arabidopsis plants, the amount of $\mathrm{M}$ trimers is decreasing when the grow-light intensity is increased from 100 to $800 \mu \mathrm{mol}$ photons $\mathrm{m}^{-2} \mathrm{~s}^{-1}$, whereas the amount of "extra" trimers remains the same. Decreasing on the other hand the intensity to $20 \mu \mathrm{mol}$ photons $\mathrm{m}^{-2} \mathrm{~s}^{-1}$, leads to an increase in the amount of "extra" trimers, whereas the amount of $\mathrm{M}$ trimers now remains unaltered (Kouril et al. 2012). For nearly all time-resolved studies in the literature, detailed information about the antenna composition is lacking.

In the past, various studies have been performed on BBY preparations (Berthold et al. 1981). The kinetics of these membranes were for instance described by a single lifetime of 210 ps (Schilstra et al. 1999) or with a major lifetime of $140 \mathrm{ps}$ and a minor lifetime of $330 \mathrm{ps}$ (Van Mieghem et al. 1992). More recently, two studies were done that showed average lifetimes in the order of 150-160 ps (Broess et al. 2006, 2008) and the results were interpreted with a coarse-grained model that uses the C2S2M2 structure as a basis. Like in the ERPE model, it was assumed that primary charge separation (with rate $k_{\mathrm{CS}}$ or inverse rate/transfer time $\tau_{\mathrm{CS}}$ ) is reversible (first chargeseparated state is $\Delta G$ lower in energy than the state in which the $\mathrm{RC}$ is excited in the $Q_{\mathrm{y}}$ state). Secondary charge separation (with rate $k_{\mathrm{RP}}$ or inverse rate/transfer time $\tau_{\mathrm{RP}}$ ) was supposed to be irreversible. EET was modeled by assuming hopping to occur between neighboring (monomeric) complexes with a rate called $k_{h}$ (or inverse rate/ hopping time $\tau_{h}$ ) that was assumed to be the same for all hopping steps, whereas each rate was scaled with the number of pigments per complex. The basic difference with the earlier ERPE model is the fact that the supercomplex is used as a structural model to include EET steps and the fact that the hopping rate is not assumed to be infinitely fast. Using this model it was shown that different combinations of $\tau_{C S}$ and $\tau_{H}$ can describe the data nearly equally well (Broess et al. 2006), reminiscent of the data fitting results for core samples. Although it was not possible to extract more details about the charge transfer kinetics in the RC, it was possible to conclude that the BBY data could not be explained with published parameters for charge separation as obtained from time-resolved studies on cores by for instance Vasilliev et al. (Vassiliev et al. 2002) and Miloslavina et al. (Miloslavina et al. 2006) and other studies. Good resemblance could only be obtained when both the rate of charge separation and the drop in free energy upon charge separation were increased. It was also argued that previously published results on isolated PSII RC (Andrizhiyevskaya et al. 2004; Groot et al. 2005) were not in accordance with the BBY results. Again, this seems to indicate that the antenna system is important for plant PSII integrity and functionality.

In a follow-up study (Broess et al. 2008), time-resolved fluorescence measurements were performed on PSII 
membranes using two different excitation wavelengths, 420 and $483 \mathrm{~nm}$. In this way, the relative number of excitations in core and outer antenna was varied, and the migration time from outer antenna to core was estimated to be 20-25 ps, much faster than one might expect based on earlier results on random aggregates of LHCII (Barzda et al. 2001). Therefore, it seems that the organization of the light-harvesting complexes in the supercomplexes/PSII membranes has been optimized in such a way that efficient EET takes place. However, at the moment detailed EET calculations are still lacking.

\section{Energy transfer and charge separation in PSII in the thylakoid membrane}

Isolated thylakoid membranes contain all complexes participating in the light reactions of photosynthesis but the large heterogeneity of the system and the presence of different complexes strongly complicate the interpretation of the time-resolved data. In general, the kinetics of thylakoids with open RCs are multi-exponential with lifetimes ranging from tens of picoseconds to values between 300 and $600 \mathrm{ps}$, and the average lifetime typically ranges from 300 to 400 ps (Engelmann et al. 2005; Leibl et al. 1989; Roelofs et al. 1992; Vasil'ev et al. 1998). However, interpretation of the individual lifetimes has remained ambiguous (for an overview see also (van Grondelle et al. 1994; Van Amerongen et al. 2003)). Recently, thylakoid membranes from A. thaliana with 4 LHCII trimers per RC were studied using various detection wavelengths to discriminate between PSI and PSII kinetics. Making use of two excitation wavelengths, it was possible to estimate the migration time from PSII outer antenna to core (van Oort et al. 2010). The fluorescence decay could be fitted very well with three lifetimes, in this particular case being 73 , 251 , and 531 ps (plus a very small contribution of a ns component) at all wavelengths with varying amplitudes. Shorter lifetimes mainly reflect spectral equilibration within individual complexes (see above) and are of less interest for the entire membrane. The three main lifetimes are sufficient to describe the data although they do not directly correspond to well-defined physical processes, and they are the result of different processes and heterogeneity in the membrane. Note that these lifetimes usually differ for different preparations, depending on for instance growth-light conditions and the state of the membrane (light- or dark-adapted, state 1 or state 2 , in the presence or absence of nonphotochemical quenching (NPQ) and with open or closed RCs). The shortest of these three lifetimes (fitted with $73 \mathrm{ps}$ in this case) is partly due to PSI whereas the other two are almost exclusively due to PSII as can be concluded from the shapes of the decay-associated spectra (van Oort et al. 2010). In this particular case, the thylakoids were dark-adapted and therefore in state 1 (no LHCII attached to PSI) with open RCs and in the absence of NPQ. It was estimated that $\tau_{\text {trap }}=180 \mathrm{ps}$ and $\tau_{\text {mig }}=150 \mathrm{ps}$. This migration time is a factor of 4-5 longer than for the PSII membranes above, which contained 2.4-2.5 trimers per RC. Therefore, it is clear that the extra trimers are connected less well to the RCs. These results indicate that at the level of the thylakoid membrane trap-limited models are certainly not valid. At this point, it is also worth mentioning that different supercomplexes are functionally connected to each other and the domain size (how far does/ can an excitation travel?) was estimated to be 12-24 LHCII trimers by Lambrev et al.(Lambrev et al. 2011).

In (Wientjes et al. 2013) it was studied for A. thaliana how the time-resolved fluorescence kinetics depends on the distribution of LHCII over PSI and PSII. In most light conditions some LHCII is attached to PSI (at most one LHCII trimer per PSI, on average around half a trimer). PSI and PSI-LHCII contribute only to the fastest ( $87 \mathrm{ps}$ in this study) component to which also PSII contributes. Lifetimes of 0.26 and $0.54 \mathrm{~ns}$ are due to PSII and are very similar to the lifetimes reported above, namely 0.25 , and $0.53 \mathrm{~ns}$ (van Oort et al. 2010) The longest lifetime is only observed in the presence of "extra" LHCII and is for instance not found for supercomplexes or PSII membranes with only 2.5 LHCII trimers per RC (see above). Upon relocation of LHCII from PSII to PSI the relative amplitude of the $87 \mathrm{ps}$ component increases at the expense of the 0.26 and $0.54 \mathrm{~ns}$ components. This is explained by a decreased contribution of the "extra" LHCIIs to the "slow" PSII fluorescence decay, and an increased contribution to the $\sim 87$ ps component by PSI-LHCII, thereby shortening the average fluorescence lifetime of the thylakoids.

\section{Where to go?}

At the level of the individual pigment-protein complexes the functioning of the outer light-harvesting complexes of PSII seems to be relatively well understood ("Outer antenna complexes" section). When it comes to the PSII core, there is more uncertainty ("The PSII core" section, ). Different labs are able to obtain very similar experimental results on the same samples but there is strong disagreement about the interpretation. Moreover, there seem to be differences between the "performance" of core complexes in vitro and in vivo and striking differences exist between core preparations from plants and cyanobacteria, although it is generally assumed that these cores are very similar. However, the cores in plants are surrounded by outer lightharvesting complexes, which is not the case in cyanobacteria. It is clear from the work on PSII supercomplexes that 
the intrinsic performance of the core of PSII is improving when the supercomplexes increase in size ("PSII supercomplexes" section). For PSII membranes, the performance seems to be even better and one might wonder to which extent the stacking of the membranes in the grana contributes to the overall performance. It is for instance still unknown how efficient EET between different membrane layers is: At the moment, the existing models mainly include EET within individual layers. It should, however, be noted that studies of Kirchhoff et al. (Kirchhoff et al. 2004) and Lambrev et al. (Lambrev et al. 2011) suggested that unstacking of the different membrane layers has no noticeable effect on excitation energy transfer, thereby implying that transfer between membrane layers is not very important. The modeling is not very sophisticated yet, which is partly due to the fact that also the structural models are not very accurate and good models should somehow also incorporate the structural variability of the membranes (in addition to heterogeneity): membranes are dynamic systems.

In thylakoid membranes where the average number of LHCII trimers can go up to four, depending on light conditions, the migration time is considerably slower, demonstrating that on the thylakoid level the charge separation process is definitely not trap-limited. It is still not known where the extra antenna complexes are located, but it is also not known to which extent they are disconnected and to which extent these complexes are quenched. There is a clear need for further studies on the grana organization and composition in different (light) conditions to enable more detailed modeling studies.

Finally, it will be very important to perform timeresolved studies in vivo, preferably at the single chloroplast level, using microscopic techniques. Only then will it be possible to see the "real" photosynthesis in action; after all, it is a very flexible and dynamic process and the chloroplast is continuously adapting to changing conditions.

Acknowledgments We thank Lijin Tian for providing Fig. 3. RC is supported by the ERC starting/consolidator Grant number 281341 and by the Netherland Organization for Scientific Research (NWO) via a Vici Grant.

Open Access This article is distributed under the terms of the Creative Commons Attribution License which permits any use, distribution, and reproduction in any medium, provided the original author(s) and the source are credited.

\section{References}

Albertsson PA, Andersson B, Larsson C, Akerlund HE (1981) Phase partition-a method for purification and analysis of cell organelles and membrane vesicles. Methods Biochem Anal 28: $115-150$
Amunts A, Toporik H, Borovikova A, Nelson N (2010) Structure determination and improved model of plant photosystem I. J Biol Chem 285(5):3478-3486

Anderson JM, Andersson B (1988) The dynamic photosynthetic membrane and regulation of solar-energy conversion. Trends Biochem Sci 13(9):351-355

Anderson JM, Chow WS, De Las Rivas J (2008) Dynamic flexibility in the structure and function of photosystem II in higher plant thylakoid membranes: the grana enigma. Photosynth Res 98(1-3): 575-587

Andersson B, Anderson JM (1980) Lateral heterogeneity in the distribution of chlorophyll-protein complexes of the thylakoid membranes of spinach chloroplasts. Biochim Biophys Acta 593: $427-440$

Andrizhiyevskaya EG, Frolov D, van Grondelle R, Dekker JP (2004) On the role of the CP47 core antenna in the energy transfer and trapping dynamics of photosystem II. Phys Chem Chem Phys 6(20):4810-4819. doi:10.1039/b411977k

Bailey S, Walters RG, Jansson S, Horton P (2001) Acclimation of Arabidopsis thaliana to the light environment: the existence of separate low light and high light responses. Planta 213(5): 794-801

Ballottari M, Mozzo M, Croce R, Morosinotto T, Bassi R (2009) Occupancy and functional architecture of the pigment binding sites of photosystem II antenna complex Lhcb5. J Biol Chem 284(12):8103-8113

Barber J (2002) Photosystem II: a multisubunit membrane protein that oxidises water. Curr Opin Struct Biol 12(4):523-530

Barzda V, Peterman EJG, van Grondelle R, Van Amerongen H (1998) The influence of aggregation on triplet formation in lightharvesting chlorophyll $a / b$ pigment-protein complex II of green plants. Biochemistry 37:546-551

Barzda V, Gulbinas V, Kananavicius R, Cervinskas V, Van Amerongen H, van Grondelle R, Valkunas L (2001) Singlet-singlet annihilation kinetics in aggregates and trimers of LHCII. BiophysJ 80(5):2409-2421

Bassi R, Sandona D, Croce R (1997) Novel aspects of chlorophyll a/ $b$-binding proteins. Physiol Plantarum 100:769-779

Bassi R, Croce R, Cugini D, Sandona D (1999) Mutational analysis of a higher plant antenna protein provides identification of chromophores bound into multiple sites. Proc Natl Acad Sci USA 96:10056-10061

Belgio E, Johnson MP, Juric S, Ruban AV (2012) Higher plant photosystem II light-harvesting antenna, not the reaction center, determines the excited-state lifetime-both the maximum and the nonphotochemically quenched. Biophys J 102(12):2761-2771. doi:10.1016/j.bpj.2012.05.004

Berthold DA, Babcock GT, Yocum CF (1981) A highly resolved, oxygen-evolving photosystem II preparation from spinach thylakoid membranes. EPR and electron-transport properties. FEBS Lett 134:231-234

Betterle N, Ballottari M, Zorzan S, de Bianchi S, Cazzaniga S, Dall'Osto L, Morosinotto T, Bassi R (2009) Light-induced dissociation of an antenna hetero-oligomer is needed for nonphotochemical quenching induction. J Biol Chem 284(22): $15255-15266$

Boekema EJ, van Breemen JF, van Roon H, Dekker JP (2000) Arrangement of photosystem II supercomplexes in crystalline macrodomains within the thylakoid membrane of green plant chloroplasts. J Mol Biol 301(5):1123-1133

Broess K, Trinkunas G, van der Weij-de Wit CD, Dekker JP, van Hoek A, van Amerongen H (2006) Excitation energy transfer and charge separation in photosystem II membranes revisited. Biophys J 91(10):3776-3786

Broess K, Trinkunas G, van Hoek A, Croce R, van Amerongen H (2008) Determination of the excitation migration time in 
photosystem II-consequences for the membrane organization and charge separation parameters. Biochim Biophys Acta 1777(5): 404-409

Caffarri S, Croce R, Breton J, Bassi R (2001) The major antenna complex of photosystem II has a xanthophyll binding site not involved in light harvesting. J Biol Chem 276(38):35924-35933

Caffarri S, Passarini F, Bassi R, Croce R (2007) A specific binding site for neoxanthin in the monomeric antenna proteins CP26 and CP29 of Photosystem II. FEBS Lett 581(24):4704-4710

Caffarri S, Kouril R, Kereiche S, Boekema EJ, Croce R (2009) Functional architecture of higher plant photosystem II supercomplexes. Embo J 28:3052-3063

Caffarri S, Broess K, Croce R, van Amerongen H (2011) Excitation energy transfer and trapping in higher plant Photosystem II complexes with different antenna sizes. Biophys J 100(9): 2094-2103. doi:10.1016/j.bpj.2011.03.049

Calhoun TR, Ginsberg NS, Schlau-Cohen GS, Cheng YC, Ballottari M, Bassi R, Fleming GR (2009) Quantum coherence enabled determination of the energy landscape in light-harvesting complex II. J Phys Chem B 113(51):16291-16295

Carbonera D, Giacometti G, Agostini G, Angerhofer A, Aust V (1992) ODMR of carotenoid and chlorophyll triplets in CP43 and CP47 complexes of spinach. Chem Phys Lett 194:275-281

Chuartzman SG, Nevo R, Shimoni E, Charuvi D, Kiss V, Ohad I, Brumfeld V, Reich Z (2008) Thylakoid membrane remodeling during state transitions in Arabidopsis. Plant Cell 20(4):1029-1039

Cinque G, Croce R, Holzwarth AR, Bassi R (2000) Energy transfer among CP29 chlorophylls: calculated Förster rates and experimental transient absorption at room temperature. BiophysJ 79:1706-1717

Clayton RK (1981) Photosynthesis: physical mechanism and chemical patterns. Cambridge University Press, Cambridge

Collini E, Scholes GD (2009) Coherent intrachain energy migration in a conjugated polymer at room temperature. Science 323(5912): 369-373. doi:10.1126/science.1164016

Connelly JP, Muller MG, Hucke M, Gatzen G, Mullineaux CW, Ruban AV, Horton P, Holzwarth AR (1997) Ultrafast spectroscopy of trimeric light harvesting complex II from higher plants. J Phys Chem B 101:1902-1909

Croce R, van Amerongen H (2011) Light-harvesting and structural organization of photosystem II: from individual complexes to thylakoid membrane. J Photochem Photobiol B 104(1-2): 142-153. doi:10.1016/j.jphotobiol.2011.02.015

Croce R, Remelli R, Varotto C, Breton J, Bassi R (1999) The neoxanthin binding site of the major light harvesting complex (LHC II) from higher plants. FEBS Lett 456:1-6

Croce R, Muller MG, Bassi R, Holzwarth AR (2001) Carotenoid-tochlorophyll energy transfer in recombinant major light- harvesting complex (LHCII) of higher plants I. Femtosecond transient absorption measurements. Biophys J 80(2):901-915

Croce R, Muller MG, Bassi R, Holzwarth AR (2003a) Chlorophyll $b$ to chlorophyll $a$ energy transfer kinetics in the CP29 antenna complex: a comparative femtosecond absorption study between native and reconstituted proteins. Biophys J 84(4):2508-2516

Croce R, Muller MG, Caffarri S, Bassi R, Holzwarth AR (2003b) Energy transfer pathways in the minor antenna complex CP29 of photosystem II: a femtosecond study of carotenoid to chlorophyll transfer on mutant and WT complexes. Biophys J 84(4): 2517-2532

Daum B, Nicastro D, Austin J II, McIntosh JR, Kuhlbrandt W (2010) Arrangement of photosystem II and ATP synthase in chloroplast membranes of spinach and pea. Plant Cell 22(4):1299-1312

de Bianchi S, Dall'Osto L, Tognon G, Morosinotto T, Bassi R (2008) Minor antenna proteins CP24 and CP26 affect the interactions between photosystem II subunits and the electron transport rate in grana membranes of arabidopsis. Plant Cell 20(4):1012-1028
Dekker JP, Boekema EJ (2005) Supramolecular organization of thylakoid membrane proteins in green plants. Biochim Biophys Acta 1706:12-39

Dunahay TG, Staehelin LA, Seibert M, Ogilvie PD, Berg SP (1984) Structural, biochemical and biophysical characterization of four oxygen-evolving photosystem II preparations from spinach. Biochim Biophys Acta 764:179-193

Durrant JR, Hastings G, Joseph DM, Barber J, Porter G, Klug DR (1992) Subpicosecond equilibration of excitation energy in isolated photosystem II reaction centers. Proc Natl Acad Sci USA 89:11632-11636

Engelmann ECM, Zucchelli G, Garlaschi FM, Casazza AP, Jennings RC (2005) The effect of outer antenna complexes on the photochemical trapping rate in barley thylakoid photosystem II. Biochim Biophys Acta 1706(3):276-286

Georgakopoulou S, van der Zwan G, Bassi R, van Grondelle R, van Amerongen H, Croce R (2007) Understanding the changes in the circular dichroism of light harvesting complex II upon varying its pigment composition and organization. Biochemistry 46(16): $4745-4754$

Germano M, Gradinaru CC, Shkuropatov AY, van Stokkum IH, Shuvalov VA, Dekker JP, van Grondelle R, van Gorkom HJ (2004) Energy and electron transfer in photosystem II reaction centers with modified pheophytin composition. Biophys J 86(3): $1664-1672$

Goral TK, Johnson MP, Brain APR, Kirchhoff H, Ruban AV, Mullineaux CW (2010) Visualizing the mobility and distribution of chlorophyll proteins in higher plant thylakoid membranes: effects of photoinhibition and protein phosphorylation. Plant $\mathrm{J}$ 62(6):948-959

Gradinaru CC, Pascal AA, van Mourik F, Robert B, Horton P, van Grondelle R, Van Amerongen H (1998) Ultrafast evolution of the excited states in the chlorophyll $a / b$ complex CP29 from green plants studied by energy-selective pump- probe spectroscopy. Biochemistry 37:1143-1149

Gradinaru CC, van Stokkum IHM, Pascal AA, van Grondelle R, Van Amerongen H (2000) Identifying the pathways of energy transfer between carotenoids and chlorophylls in LHCII and CP29. A multicolor, femtosecond pump-probe study. J Phys Chem B 104: 9330-9342

Green BR, Khlbrandt W (1995) Sequence conservation of lightharvesting and stress- response proteins in relation to the threedimensional molecular structure of LHCII. Photosynth Res 44: 139-148

Groot ML, Pawlowicz NP, van Wilderen LJGW, Breton J, van Stokkum IHM, van Grondelle R (2005) Initial electron donor and acceptor in isolated photosystem II reaction centers identified with femtosecond mid-IR spectroscopy. Proc Natl Acad Sci USA 102(37):13087-13092

Guskov A, Kern J, Gabdulkhakov A, Broser M, Zouni A, Saenger W (2009) Cyanobacterial photosystem II at 2.9-angstrom resolution and the role of quinones, lipids, channels and chloride. Nat Struct Mol Biol 16(3):334-342

Hankamer B, Nield J, Zheleva D, Boekema E, Jansson S, Barber J (1997) Isolation and biochemical characterisation of monomeric and dimeric photosystem II complexes from spinach and their relevance to the organisation of photosystem II in vivo. Eur $\mathbf{J}$ Biochem 243:422-429

Holzwarth AR, Muller MG, Reus M, Nowaczyk M, Sander J, Rogner $M$ (2006) Kinetics and mechanism of electron transfer in intact photosystem II and in the isolated reaction center: pheophytin is the primary electron acceptor. Proc Natl Acad Sci USA 103(18): 6895-6900

Jahns P, Holzwarth AR (2012) The role of the xanthophyll cycle and of lutein in photoprotection of photosystem II. Biochim Biophys Acta 1817(1):182-193. doi:10.1016/j.bbabio.2011.04.012 
Kereiche S, Kiss AZ, Kouril R, Boekema EJ, Horton P (2010) The PsbS protein controls the macro-organisation of photosystem II complexes in the grana membranes of higher plant chloroplasts. FEBS Lett 584(4):759-764

Kirchhoff H, Borinski M, Lenhert S, Chi LF, Buchel C (2004) Transversal and lateral exciton energy transfer in grana thylakoids of spinach. Biochemistry 43(45):14508-14516

Kirchhoff H, Haase W, Wegner S, Danielsson R, Ackermann R, Albertsson PA (2007) Low-light-induced formation of semicrystalline photosystem II arrays in higher plant chloroplasts. Biochemistry 46(39):11169-11176

Kiss AZ, Ruban AV, Horton P (2008) The PsbS protein controls the organization of the photosystem II antenna in higher plant thylakoid membranes. J Biol Chem 283(7):3972-3978

Kobayashi M, Ohashi S, Iwamoto K, Shiraiwa Y, Kato Y, Watanabe $\mathrm{T}$ (2007) Redox potential of chlorophyll d in vitro. Biochim Biophys Acta 1767(6):596-602

Kouril R, Oostergetel GT, Boekema EJ (2011) Fine structure of granal thylakoid membrane organization using cryo electron tomography. Biochim Biophys Acta 1807(3):368-374. doi: 10.1016/j.bbabio.2010.11.007

Kouril R, Wientjes E, Bultema JB, Croce R, Boekema EJ (2012) High-light vs. low-light: effect of light acclimation on photosystem II composition and organization in Arabidopsis thaliana. Biochim Biophys Acta 1827(3):411-419. doi:10.1016/j.bbabio. 2012.12.003

Kovacs L, Damkjaer J, Kereiche S, Ilioaia C, Ruban AV, Boekema EJ, Jansson S, Horton P (2006) Lack of the light-harvesting complex $\mathrm{CP} 24$ affects the structure and function of the grana membranes of higher plant chloroplasts. Plant Cell 18(11):3106-3120

Kwa SLS, Groeneveld FG, Dekker JP, van Grondelle R, Van Amerongen H, Lin S, Struve WS (1992) Steady-state and timeresolved polarized light spectroscopy of the green plant lightharvesting complex II. Biochim Biophys Acta 1101:143-146

Lambrev PH, Schmitt FJ, Kussin S, Schoengen M, Varkonyi Z, Eichler HJ, Garab G, Renger G (2011) Functional domain size in aggregates of light-harvesting complex II and thylakoid membranes. Biochim Biophys Acta 1807(9):1022-1031. doi:10.1016/ j.bbabio.2011.05.003

Lampoura SS, Barzda V, Owen GM, Hoff AJ, Van Amerongen H (2002) Aggregation of LHCII leads to a redistribution of the triplets over the central xanthophylls in LHCII. Biochemistry 41(29): 9139-9144

Leibl W, Breton J, Deprez J, Trissl HW (1989) Photoelectric study on the kinetics of trapping and charge stabilization in oriented PS II membranes. Photosynth Res 22:257-275

Liu Z, Yan H, Wang K, Kuang T, Zhang J, Gui L, An X, Chang W (2004) Crystal structure of spinach major light-harvesting complex at 2.72 A resolution. Nature 428(6980):287-292

Marin A, Passarini F, Croce R, van Grondelle R (2010) Energy transfer pathways in the $\mathrm{CP} 24$ and $\mathrm{CP} 26$ antenna complexes of higher plant photosystem II: a comparative study. Biophys J 99(12): 4056-4065

Marin A, Passarini F, van Stokkum IH, van Grondelle R, Croce R (2011) Minor complexes at work: light-harvesting by carotenoids in the photosystem II antenna complexes CP24 and CP26. Biophys J 100(11):2829-2838. doi:10.1016/j.bpj.2011.04.029

Miloslavina Y, Szczepaniak M, Muller MG, Sander J, Nowaczyk M, Rogner M, Holzwarth AR (2006) Charge separation kinetics in intact photosystem II core particles is trap-limited. A picosecond fluorescence study. Biochemistry 45(7):2436-2442

Minagawa J, Takahashi Y (2004) Structure, function and assembly of photosystem II and its light-harvesting proteins. Photosynth Res 82(3):241-263

Mozzo M, Dall'Osto L, Hienerwadel R, Bassi R, Croce R (2008a) Photoprotection in the antenna complexes of photosystem II- role of individual xanthophylls in chlorophyll triplet quenching. J Biol Chem 283(10):6184-6192

Mozzo M, Passarini F, Bassi R, van Amerongen H, Croce R (2008b) Photoprotection in higher plants: the putative quenching site is conserved in all outer light-harvesting complexes of photosystem II. Biochim Biophys Acta 1777(10):1263-1267

Muh F, Renger T (2012) Refined structure-based simulation of plant light-harvesting complex II: linear optical spectra of trimers and aggregates. Biochim Biophys Acta 1817(8):1446-1460. doi: 10.1016/j.bbabio.2012.02.016

Muh F, Renger T, Zouni A (2008) Crystal structure of cyanobacterial photosystem II at 3.0 angstrom resolution: a closer look at the antenna system and the small membrane-intrinsic subunits. Plant Physiol Biochem 46(3):238-264

Mustardy L, Garab G (2003) Granum revisited. A three-dimensional model-where things fall into place. Trends Plant Sci 8(3):117-122

Mustardy L, Buttle K, Steinbach G, Garab G (2008) The threedimensional network of the thylakoid membranes in plants: quasihelical model of the granum-stroma assembly. Plant Cell 20(10):2552-2557

Neilson JA, Durnford DG (2010) Evolutionary distribution of lightharvesting complex-like proteins in photosynthetic eukaryotes. Genome 53(1):68-78

Nelson N, Yocum CF (2006) Structure and function of photosystems I and II. Annu Rev Plant Biol 57:521-565

Novoderezhkin VI, van Grondelle R (2010) Physical origins and models of energy transfer in photosynthetic light-harvesting. Phys Chem Chem Phys 12(27):7352-7365

Novoderezhkin V, Palacios MA, Van Amerongen H, van Grondelle R (2004) Energy-transfer dynamics in the LHCII complex of higher plants: modified redfield approach. J Phys Chem B 108(29):10363-10375

Novoderezhkin VI, Palacios MA, Van Amerongen H, van Grondelle R (2005) Excitation dynamics in the LHCII complex of higher plants: modeling based on the 2.72 angstrom crystal structure. J Phys Chem B 109(20):10493-10504

Palacios MA, Standfuss J, Vengris M, van Oort BF, van Stokkum IH, Kuhlbrandt W, van Amerongen H, van Grondelle R (2006) A comparison of the three isoforms of the light-harvesting complex II using transient absorption and time-resolved fluorescence measurements. Photosynth Res 88(3):269-285

Pan X, Li M, Wan T, Wang L, Jia C, Hou Z, Zhao X, Zhang J, Chang W (2011) Structural insights into energy regulation of lightharvesting complex CP29 from spinach. Nat Struct Mol Biol 18(3):309-315. doi:10.1038/nsmb.2008

Pascal A, Gradinaru C, Wacker U, Peterman E, Calkoen F, Irrgang KD, Horton P, Renger G, van Grondelle R, Robert B, Van Amerongen H (1999) Spectroscopic characterization of the spinach Lhcb4 protein (CP29), a minor light-harvesting complex of photosystem II. Eur J Biochem 262:817-823

Passarini F, Wientjes E, Hienerwadel R, Croce R (2009) Molecular basis of light harvesting and photoprotection in CP24 Unique Features of the most recent antenna complex. J Biol Chem 284(43): 29536-29546

Pawlowicz NP, van Grondelle R, van Stokkum IH, Breton J, Jones MR, Groot ML (2008) Identification of the first steps in charge separation in bacterial photosynthetic reaction centers of Rhodobacter sphaeroides by ultrafast mid-infrared spectroscopy: electron transfer and protein dynamics. Biophys J 95(3): $1268-1284$

Peterman EJG, Hobe S, Calkoen F, van Grondelle R, Paulsen H, van Amerongen H (1996) Low-temperature spectroscopy of monomeric and trimeric forms of reconstituted light-harvesting chlorophyll a/b complex. Biochim Biophys Acta 1273:171-174

Peterman EJG, Monshouwer R, van Stokkum IHM, van Grondelle R, Van Amerongen H (1997) Ultrafast singlet excitation transfer from 
carotenoids to chlorophylls via different pathways in light-harvesting complex II of higher plants. Chem Phys Lett 264:279-284

Plumley FG, Schmidt GW (1987) Reconstitution of chloroform $a / b$ light-harvesting complexes: xanthophyll-dependent assembley and energy transfer. Proc Natl Acad Sci USA 84:146-150

Prokhorenko VI, Holzwarth AR (2000) Primary process and structure of the photosystem II reaction center: a photon echo study. J Phys Chem B 104:11563-11578

Rappaport F, Boussac A, Force DA, Peloquin J, Brynda M, Sugiura M, Un S, Britt RD, Diner BA (2009) Probing the coupling between proton and electron transfer in photosystem II core complexes containing a 3-fluorotyrosine. J Am Chem Soc 131(12):4425-4433

Raszewski G, Renger T (2008) Light harvesting in photosystem II core complexes is limited by the transfer to the trap: can the core complex turn into a photoprotective mode? J Am Chem Soc 130(13):4431-4446

Remelli R, Varotto C, Sandona D, Croce R, Bassi R (1999) Chlorophyll binding to monomeric light-harvesting complex. A mutation analysis of chromophore-binding residues. J Biol Chem 274(47):33510-33521

Renger G (2010) The light reactions of photosynthesis. Curr Sci 98(10):1305-1319

Renger G, Renger T (2008) Photosystem II: the machinery of photosynthetic water splitting. Photosynth Res 98(1-3):53-80

Renger T, Schlodder E (2010) Primary photophysical processes in photosystem II: bridging the gap between crystal structure and optical spectra. Chem Phys Chem 11(6):1141-1153

Roelofs TA, Lee CH, Holzwarth AR (1992) Global target analysis of picosecond chlorophyll fluorescence kinetics from pea chloroplasts. A new approach to the characterization of the primary processes in photosystem II alfa- and beta-units. Biophys $\mathrm{J}$ 61: $1147-1163$

Rogl H, Kuhlbrandt W (1999) Mutant trimers of light-harvesting complex II exhibit altered pigment content and spectroscopic features. Biochemistry 38(49):16214-16222

Ruban AV, Horton P (1999) The xanthophyll cycle modulates the kinetics of nonphotochemical energy dissipation in isolated light-harvesting complexes, intact chloroplasts, and leaves of spinach. Plant Physiol 119:531-542

Salverda JM, Vengris M, Krueger BP, Scholes GD, Czarnoleski AR, Novoderezhkin V, Van Amerongen H, van Grondelle R (2003) Energy transfer in light-harvesting complexes LHCII and CP29 of spinach studied with three pulse echo peak shift and transient grating. BiophysJ 84(1):450-465

Sandona D, Croce R, Pagano A, Crimi M, Bassi R (1998) Higher plants light harvesting proteins. Structure and function as revealed by mutation analysis of either protein or chromophore moieties. Biochim Biophys Acta 1365:207-214

Savikhin S, Van Amerongen H, Kwa SLS, van Grondelle R, Struve WR (1994a) Low-temperature energy transfer in LHC-II trimers from the Chl $a / b$ light-harvesting antenna of photosystem II. BiophysJ 66:1597-1603

Savikhin S, Zhu YW, Lin S, Blankenship RE, Struve WS (1994b) Femtosecond spectroscopy of chlorosome antennas from the green photosynthetic bacterium chloroflexus aurantiacus. J Phys Chem 98:10322-10334

Schatz GH, Brock H, Holzwarth AR (1987) Picosecond kinetics of fluorescence and absorbance changes in photosystem II particles excited at low photon density. Proc Natl Acad SciUSA 84: 8414-8418

Schatz GH, Brock H, Holzwarth AR (1988) A kinetic and energetic model for the primary processes in photosystem II. Biophys J 54:397-405

Schilstra MJ, Nield J, Dorner W, Hankamer B, Carradus M, Barter LMC, Barber J, Klug DR (1999) Similarity between electron donor side reactions in the solubilized photosystem II-LHC II supercomplex and photosystem-II-containing membranes. Photosynth Res 60(2-3):191-198

Shimoni E, Rav-Hon O, Ohad I, Brumfeld V, Reich Z (2005) Threedimensional organization of higher-plant chloroplast thylakoid membranes revealed by electron tomography. Plant Cell 17(9): $2580-2586$

Standfuss R, van Scheltinga ACT, Lamborghini M, Kuhlbrandt W (2005) Mechanisms of photoprotection and nonphotochemical quenching in pea light-harvesting complex at $2.5 \mathrm{~A}$ resolution. EMBO J 24(5):919-928

Tian L, van Stokkum IH, Koehorst RB, Jongerius A, Kirilovsky D, van Amerongen $H$ (2011) Site, rate, and mechanism of photoprotective quenching in cyanobacteria. J Am Chem Soc 133(45):18304-18311. doi:10.1021/ja206414m

Tian L, van Stokkum IH, Koehorst RB, van Amerongen H (2012) Light harvesting and blue-green light induced non-photochemical quenching in two different C-phycocyanin mutants of synechocystis PCC 6803. J Phys Chem. doi:10.1021/jp309570u

Tian L, Farooq S, van Amerongen H (2013) Probing the picosecond kinetics of the photosystem II core complex in vivo. Phys Chem Chem Phys. doi:10.1039/c3cp43813a

Umena Y, Kawakami K, Shen JR, Kamiya N (2011) Crystal structure of oxygen-evolving photosystem II at a resolution of $1.9 \mathrm{~A}$. Nature 473(7345):55-60. doi:10.1038/nature09913

Van Amerongen H, van Grondelle R (2001) Understanding the energy transfer function of LHCII, the major light-harvesting complex of green plants. J Phys Chem B 105(3):604-617

Van Amerongen H, Kwa SLS, van Bolhuis BM, van Grondelle R (1994) Polarized fluorescence and absorption of macroscopically aligned light harvesting complex II. Biophys J 67:837-847

Van Amerongen H, Valkunas L, van Grondelle R (2000) Photosynthenic excitons. World Scientific Publishing Co. Pte. Ltd, Singapore

Van Amerongen H, Dekker JP, Parson WW, Green BR (2003) Lightharvesting antennas in photosynthesis. Kluwer Academic, The Netherlands, pp 219-251

van der Vos R, Carbonera D, Hoff AJ (1991) Microwave and optical spectroscopy of carotenoid triplets in light-harvesting complex LHCII of spinach by absorbance-detected magnetic resonance. J Appl Magn Reson 2:179-202

van der Weij-de Wit CD, Dekker JP, van Grondelle R, van Stokkum IH (2011) Charge separation is virtually irreversible in photosystem II core complexes with oxidized primary quinone acceptor. J Phys Chem A 115(16):3947-3956. doi:10.1021/jp108 3746

van Grondelle R (1985) Excitation energy transfer, trapping and annihilation in photosynthetic systems. Biochim Biophys Acta 811:147-195

van Grondelle R, Dekker JP, Gillbro T, Sundstr"m V (1994) Energy transfer and trapping in photosynthesis. Biochim Biophys Acta 1187:1-65

Van Mieghem FJE, Searle GFW, Rutherford AW, Schaafsma TJ (1992) The influence of the double reduction of $\mathrm{Q}(\mathrm{A})$ on the fluorescence decay kinetics of photosystem II. Biochim Biophys Acta 1100:198-206

van Mourik F, Groot ML, van Grondelle R, Dekker JP, van Stokkum IHM (2004) Global and target analysis of fluorescence measurements on photosystem 2 reaction centers upon red excitation. Phys Chem Chem Phys 6(20):4820-4824

van Oort B, van Hoek A, Ruban AV, van Amerongen H (2007) Equilibrium between quenched and nonquenched conformations of the major plant light-harvesting complex studied with highpressure time-resolved fluorescence. J Phys Chem 111(26): 7631-7637

van Oort B, Alberts M, de Bianchi S, Dall'Osto L, Bassi R, Trinkunas G, Croce R, van Amerongen H (2010) Effect of antenna- 
depletion in photosystern II on excitation energy transfer in Arabidopsis thaliana. Biophys J 98(5):922-931

Vasil'ev S, Wiebe S, Bruce D (1998) Non-photochemical quenching of chlorophyll fluorescence in photosynthesis. 5-Hydroxy-1,4naphthoquinone in spinach thylakoids as a model for antenna based quenching mechanisms. Biochim Biophys Acta 1363: $147-156$

Vassiliev S, Bruce D (2008) Toward understanding molecular mechanisms of light harvesting and charge separation in photosystem II. Photosynth Res 97(1):75-89

Vassiliev S, Lee CI, Brudvig GW, Bruce D (2002) Structure-based kinetic modeling of excited-state transfer and trapping in histidine-tagged photosystem II core complexes from synechocystis. Biochemistry 41(40):12236-12243

Visser HM, Kleima FJ, van Stokkum IHM, van Grondelle R, Van Amerongen H (1996) Probing the many energy-transfer processes in the photosynthetic light-harvesting complex II at $77 \mathrm{~K}$ using energy-selective sub- picosecond transient absorption spectroscopy. Chem Phys 210:297-312

Wasielewski MR, Johnson DG, Govindjee Preston C, Seibert M, Baltscheffsky M (1990) The primary charge-separation rate in isolated photosystem II reaction center complex. Current research in photosynthesis. Kluwer Academic Publishers, Dordrecht, pp 451-454

Wientjes E, Oostergetel GT, Jansson S, Boekema EJ, Croce R (2009) The role of Lhca complexes in the supramolecular organization of higher plant photosystem I. J Biol Chem 284(12):7803-7810

Wientjes E, van Amerongen H, Croce R (2013) LHCII is an antenna of both photosystems after long-term acclimation. Biochim Biophys Acta 1827(3):420-426. doi:10.1016/j.bbabio.2012. 12.009

Yakushevska AE, Jensen PE, Keegstra W, van Roon H, Scheller HV, Boekema EJ, Dekker JP (2001) Supermolecular organization of photosystem II and its associated light-harvesting antenna in Arabidopsis thaliana. Eur J Biochem 268(23):6020-6028

Yang CH, Kosemund K, Cornet C, Paulsen H (1999) Exchange of pigment-binding amino acids in light-harvesting chlorophyll $a / b$ protein. Biochemistry 38(49):16205-16213

Zucchelli G, Santabarbara S, Jennings RC (2012) The Q(y) absorption spectrum of the light-harvesting complex II as determined by structure-based analysis of chlorophyll macrocycle deformations. Biochemistry 51(13):2717-2736. doi:10.1021/bi201677q 